\title{
Claulansine F promotes neuritogenesis in PC12 cells via the ERK signaling pathway
}

\author{
Yin-zhong $\mathrm{MA}^{1,2}, \mathrm{Na} \mathrm{NING}^{1}$, Wen-bin $\mathrm{HE}^{1,3}$, Jing-wei $\mathrm{LI}^{1,2}$, Jin-feng $\mathrm{HU}^{1}$, Shi-feng $\mathrm{CHU}^{1}$, Nai-hong $\mathrm{CHEN}^{1, *}$ \\ ${ }^{1}$ State Key Laboratory of Bioactive Substances and Functions of Natural Medicines, Beijing Key Laboratory of New Drug Mechanisms \\ and Pharmacological Evaluation Study, Department of Pharmacology, Institute of Materia Medica, Chinese Academy of Medical \\ Sciences and Peking Union Medical College, Beijing 100050, China; ${ }^{2}$ Tianjin University of Traditional Chinese Medicine, Tianjin \\ 300193, China; ${ }^{3}$ Shanxi University of Traditional Chinese Medicine, Taiyuan 030024, China
}

\begin{abstract}
Aim: To study the effects of Claulansine F (Clau F), a carbazole alkaloid isolated from the stem of Clausena lansium (Lour) Skeels, on neuritogenesis of PC12 cells, and to elucidate the mechanism of action.

Methods: Neuritogenesis of PC12 cells was quantified under an inverted microscope. Expression of the neurite outgrowth marker GAP43 was detected using immunofluorescence. GAP-43 transcription was measured using RT-PCR. Cell viability was evaluated with MTT assay. The levels of phosphor-ERK1/2, phosphor-CREB, phosphor-AKT and acetylate-p53 in the cells were examined using Western blotting analyses.

Results: Clau F (10-100 $\mu \mathrm{mol} / \mathrm{L})$ significantly increased the percentage of PC12 cells bearing neurites. Clau F markedly increased the expression of GAP-43 in the cells. The efficiency of Clau F (10 $\mu \mathrm{mol} / \mathrm{L})$ in increasing neuritogenesis and GAP-43 expression was comparable to that of nerve growth factor $(50 \mathrm{ng} / \mathrm{mL})$. In addition, Clau F completely blocked the proliferation of PC12 cells within $7 \mathrm{~d}$ of incubation, whereas it did not cause cell death in cultured rat cortical neurons. Treatment of PC12 cells with Clau F activated both ERK and AKT signaling pathways. Co-treatment of PC12 cells with the specific ERK inhibitor PD98059, but not the specific PI3K inhibitor LY294002, blocked Clau F-induced neuritogenesis and GAP-43 upregulation.

Conclusion: Clau F promotes neuritogenesis in PC12 cells specifically via activation of the ERK signaling pathway.
\end{abstract}

Keywords: Claulansine F; PC12 cell; neuritogenesis; GAP-43; ERK1/2; nerve growth factor; neurodegenerative disease

Acta Pharmacologica Sinica (2013) 34: 1499-1507; doi: 10.1038/aps.2013.95; published online 7 Oct 2013

\section{Introduction}

The cause of many neurodegenerative disorders such as Alzheimer's disease, Parkinson's disease and amyotrophic lateral sclerosis can be ascribed to the loss of functional neurons $^{[1]}$. Induction of neurite outgrowth by administration of endogenous trophic factors has been proposed as one of several new therapeutic strategies ${ }^{[2]}$. However, clinical trials with neurotrophic factors have been rather disappointing because these drugs either caused tumorigenesis or failed to be delivered into the central nervous system ${ }^{[3,4]}$. Therapeutic efforts aimed at developing non-peptidic liposoluble small molecules acting on signaling pathways involved in neurite initiation have been proposed as a valid alternative or adjunct to solve these problems ${ }^{[5]}$. Such compounds could penetrate the blood-brain barrier and further promote neuritogenesis at the impaired site, thus exerting neurotrophin-like activities

\footnotetext{
* To whom correspondence should be addressed.

E-mail chennh@imm.ac.cn

Received 2013-03-29 Accepted 2013-06-24
}

without major difficulties.

In this regard, natural products are attractive sources of potentially effective drugs. Claulansine F (Clau F) is a carbazole alkaloid isolated from the stem of Clausena lansium (Lour) Skeels ${ }^{[6]}$, a member of the Rutaceae family. The structure of Clau F is shown in Figure 1. Unlike the other carbazole alkaloids which only exhibit antitumor or antifungal activity, Clau $\mathrm{F}$ inhibits cell proliferation and promotes neurite outgrowth in PC12 cells in addition. Because Clau F has a novel chemical structure according to its bioactivity, we decided to further

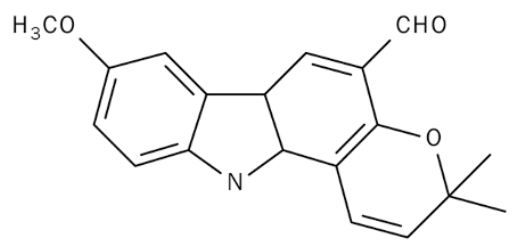

Figure 1. Structure of Clau F. 
analyze the signal transduction pathways underlying its neuritogenesis-promoting effects.

The rat PC12 pheochromocytoma cell line is a well-established cell model that differentiates into a neuronal phenotype with neurite outgrowth after exposure to neurotrophic peptides such as nerve growth factor $(\mathrm{NGF})^{[7]}$. The binding of NGF to high-affinity tyrosine kinase A (Trk A) receptors mainly triggers two cellular signaling cascades: the MAPKERK and PI3K-AKT pathways, which modulate cell proliferation and differentiation, respectively ${ }^{[8,9]}$. After binding to phosphotyrosine-containing recognition elements of TrK A receptors, signal molecules of the MAPK and inositol triphosphate (PI3) kinase pathways are phosphorylated and thus activated. The activation of PI3 kinase pathways thus triggers gene transcription and regulation of the cytoskeletal machinery. In these differentiation conditions, sustained activation of the MAPK-ERK pathway and its downstream transcription factor CREB has an essential role in cellular transduction ${ }^{[10]}$. Moreover, the acetylation of p53, which is triggered by PI3K$\mathrm{AKT}$, has also been implicated in the regulation of axonal outgrowth $^{[11]}$.

Clau F can exert a neurotrophin-like effect, which may be due to its chemical structure. However, here, we focused on observing the efficacy of Clau F with respect to its ability to promote neurite outgrowth and clarifying its underlying mechanism of action, which is implicated in the NGF-mediated differentiation process.

\section{Materials and methods}

\section{Chemicals and reagents}

Clau F was obtained as previously described ${ }^{[12]}$. NGF was purchased from the National Institute for Control of Pharmaceutical and Biological Products (Beijing, China). PD98059 and LY294002 were purchased from Cell Signaling Technology (Danvers, MA, USA). Hoechst 33342 and 3-(4,5-dimethylthiazol-2-yl)-2,5-diphenyl-tetrazolium bromide (MTT) were purchased from Sigma Chemical Company (St Louis, MO, USA). Cell membrane proteins, cytoplasmic proteins and nuclear proteins were extracted using the Nucl-Cyto-Mem Preparation Kit (Applgen, Beijing, China). Antibodies that recognize acetylated p53 were purchased from Upstate Biotechnology (Waltham, MA, USA). GAP-43 and the phosphorylated forms of ERK1/2 (Thr202/Tyr204), CREB (Ser133) and AKT (Ser473) were purchased from Santa Cruz Biotechnology (Dallas, TX, USA). All secondary antibodies were purchased from Cell Signaling Technology (Danvers, MA, USA). All other chemicals were of analytical grade and were commercially available.

\section{PC12 cell culture}

PC12 cells were purchased from the American Type Culture Collection (ATCC, USA). The cells were cultured in Dulbecco's modified Eagle's medium (DMEM; Invitrogen, Gibco, USA) supplemented with $5 \%$ heat-inactivated fetal bovine serum (FBS; Invitrogen, Gibco, USA) and 5\% equine serum (ES; Thermo Scientific, Hyclone, USA). The cultures were maintained in a humidified incubator at $37^{\circ} \mathrm{C}$ in an atmo- sphere of $95 \%$ air and $5 \% \mathrm{CO}_{2}$. The media were changed every $2 \mathrm{~d}$ during the culture.

\section{Newborn Sprague-Dawley (SD) rat cell culture}

Newborn SD rats (born less than $24 \mathrm{~h}$ prior to dissections) were purchased from Vital River Laboratory Animal Technology Co, Ltd. Cerebral cortices were dissected from the rats and cultured in DMEM/F12 medium (Invitrogen, Gibco, USA) containing $10 \%$ ES. The cultures were maintained in a humidified incubator at $37^{\circ} \mathrm{C}$ in an atmosphere of $95 \%$ air and $5 \% \mathrm{CO}_{2}$. The media were changed every $2 \mathrm{~d}$ during the culture. All animals were handled in accordance with the standards established in the Guide for the Care and Use of Laboratory Animals published by the Institute of Laboratory Animal Resources of the National Research Council (USA), and the procedures were approved by the Animal Care and Use Committee of the Peking Union Medical College and the Chinese Academy of Medical Sciences (Beijing, China). All efforts were made to minimize the number of animals used as well as their suffering.

\section{Cell viability assay}

Cells were plated in poly-L-lysine-coated $96-$ well plates in serum-free medium at a density of $5 \times 10^{3}$ cells per well and allowed to attach for $24 \mathrm{~h}$. Then, the cells were washed and incubated in DMEM supplemented with 5\% FBS, 5\% ES and $10 \mu \mathrm{mol} / \mathrm{L}$ Clau F or $50 \mathrm{ng} / \mathrm{mL}$ NGF for $4 \mathrm{~d}$. During this time, the experimental compound was renewed every $2 \mathrm{~d}$. After incubation, the cells were treated with MTT solution for $4 \mathrm{~h}$ and exposed to an MTT-formazan dissolving solution [a mixture of methanol and dimethyl sulfoxide (DMSO)]. The optical density $(O D)$ was determined using an absorbance microplate reader (Molecular Devices, Toronto, Canada) set at a wavelength of $570 \mathrm{~nm}$. The cell viability was expressed as a percentage of the $O D$ value of the control cultures.

\section{Neurite outgrowth}

Cells were plated in poly-L-lysine-coated 24-well plates in serum-free medium at a density of $5 \times 10^{3}$ cells per well and allowed to attach for $24 \mathrm{~h}$. Then, the cells were washed and incubated in DMEM supplemented with 5\% FBS, 5\% ES and $10 \mathrm{\mu mol} / \mathrm{L}$ Clau F for $6 \mathrm{~d}$. During this time, the experimental compound was renewed every $2 \mathrm{~d}$. Quantification of neurite outgrowth was performed as previously described ${ }^{[13]}$. Briefly, the cells were examined under a microscope, and only processes longer than $20 \mu \mathrm{m}$ were considered as neurites. Neurite outgrowth was quantified as the percentage of cells bearing neurites with lengths at least twice as long as the cell diameter.

\section{Immunocytochemical staining}

Cells were fixed in $4 \%$ formaldehyde, membrane-permeabilized in Triton X-100 and blocked using goat serum. The cells were then exposed to a primary antibody overnight at $4{ }^{\circ} \mathrm{C}$ followed by incubation at room temperature with an Alexa Fluor 488-conjugated secondary antibody. 


\section{Hoechst staining}

Cells were fixed in $4 \%$ formaldehyde, membrane-permeabilized in Triton X-100 and subsequently exposed to $2 \mu \mathrm{g} / \mathrm{mL}$ Hoechst 33324 dissolved in 95\% glycerin. Fluorescent images were acquired by fluorescence microscopy.

\section{Western blotting analysis}

Cells were harvested in cell lysis buffer, and protein was separated by sodium dodecyl sulfate-polyacrylamide gel electrophoresis (SDS-PAGE) and transferred onto polyvinyldifluoride membranes. After incubation overnight at $4^{\circ} \mathrm{C}$ with primary antibody, the membranes were incubated with HRPconjugated secondary antibodies. The blots were developed using an ECL Plus Kit (Amersham Biosciences, Aylesbury, UK) and exposed to Kodak autoradiographic film. All data were obtained from three independent experiments and were expressed as ratios of the $O D$ values of the corresponding controls for statistical analyses (Tukey's test).

\section{Reverse transcription PCR (RT-PCR) assay}

RT-PCR was performed to determine the mRNA expression of GAP-43 in PC12 cells. Total cellular RNA was isolated using TRIzol reagent following the manufacturer's protocols and quantified by measuring the absorbance at $260 \mathrm{~nm}$. The RNA purity was determined using the A260/ A280 ratio (average ratio $>1.85)$. Total RNA from each sample was reversetranscribed into cDNA using the Reverse Transcription System (Takara Biotechnology, Otsu, Shiga, Japan). The cDNAs were amplified with the following specific primers: GAP-43, 5'-ATGCTGTGCTGTATGAGAAG-3' (upstream); 5'-TCAGGCATGTTCTTGGTCAG-3' (downstream). Amplification was performed as follows: 35 cycles of $95^{\circ} \mathrm{C}$ for $30 \mathrm{~s}, 52^{\circ} \mathrm{C}$ for $30 \mathrm{~s}$, and $72^{\circ} \mathrm{C}$ for $45 \mathrm{~s}$. The PCR products were normalized in relation to $\beta$-actin mRNA standards.

\section{Statistical analysis}

The data are expressed as the mean \pm SEM. Analysis of variance (ANOVA) followed by Dunnett's test or Tukey's test was used for statistical comparisons. The level of statistical significance was set at $P<0.05$ and $P<0.01$.

\section{Results}

\section{Clau F promotes neurite outgrowth in PC12 cells}

First, we examined whether the pro-neuritogenesis effect of Clau F was concentration-dependent. As was shown in Figure 2A, a 10 to $100 \mu \mathrm{mol} / \mathrm{L}$ final concentration of Clau F caused significant neuritogenesis $(P<0.01)$ in PC12 cells. Although no apparent cytotoxicity was observed in the $50 \mu \mathrm{mol} / \mathrm{L}$ or 100 $\mu \mathrm{mol} / \mathrm{L}$ group, crystallization of Clau F can be observed at a 50 or $100 \mu \mathrm{mol} / \mathrm{L}$ final concentration. This is most likely due to the relatively low polarity of Clau F. Therefore, we utilized a $10 \mu \mathrm{mol} / \mathrm{L}$ final concentration in subsequent experiments.

We used NGF as a positive control in this neuronal model. The pro-differentiation potency of NGF $(50 \mathrm{ng} / \mathrm{mL})$ was comparable to that described in the literature after a 2-d treatment ${ }^{[14]}$. We next investigated the pro-neuritogenesis effect of Clau F by evaluating the synthesis of GAP-43, a marker of neurite outgrowth. PC12 cells were exposed to $10 \mu \mathrm{mol} / \mathrm{L}$ Clau $\mathrm{F}$ or $50 \mathrm{ng} / \mathrm{mL}$ NGF for $2 \mathrm{~d}$ before being fixed and subjected to GAP-43 immunostaining and Hoechst 33342 staining. We found that both agents induced a higher expression level of GAP-43 compared with the control group as shown in Figure 2B. For long-term incubation, PC12 cells were exposed to Clau F or NGF for different lengths of time ( $\leq 6 \mathrm{~d})$, and the percentage of cells with neurites was determined. As shown in Figure 2C, the percentages of cells with neurites induced by NGF and Clau F were $69.7 \% \pm 4.1 \%$ and $66.3 \% \pm 3.2 \%$, respectively, after $6 \mathrm{~d}$ of treatment, showing that the efficacy of Clau F for promoting neurite outgrowth was similar to that of NGF. The total proteins of these cells were extracted for Western blotting analysis, and total cellular RNA was isolated for RTPCR assays. As shown in Figure 2D, both agents caused a gradual increase in GAP-43 protein and mRNA levels over 7 d.

\section{Clau F inhibits cell proliferation without causing cell death}

Although NGF significantly promoted cell proliferation, Clau F was able to restrain cell proliferation based on our preliminary screen of cell viability. As shown in Figure 3A, within $7 \mathrm{~d}$ of treatment with Clau F $(10 \mu \mathrm{mol} / \mathrm{L})$, a significant inhibitory effect on cell proliferation was noted compared with the control group. To determine whether Clau F caused cell death due to cytotoxicity, we separated the cerebral cortex from newborn SD rats, dispersed the cells and treated them with Clau F $(10 \mu \mathrm{mol} / \mathrm{L})$ for $7 \mathrm{~d}$. We then evaluated the cell viability by MTT assay. Figure 3B illustrates that no significant viability change was observed among the control group at $0 \mathrm{~d}$ (a), the control group at $7 \mathrm{~d}(\mathrm{~b})$ and the Clau F treatment group at $7 \mathrm{~d}$ (c), suggesting that Clau F was not cytotoxic at a final concentration of $10 \mu \mathrm{mol} / \mathrm{L}$.

\section{Clau F activates the ERK and AKT signaling pathways in PC12 cells}

To determine whether the MAPK-ERK and PI3K-AKT signaling pathways are involved in our model, $10 \mu \mathrm{mol} / \mathrm{L}$ Clau $\mathrm{F}$ or $50 \mathrm{ng} / \mathrm{mL}$ NGF treatments were applied for different durations $(\leq 1 \mathrm{~h})$ followed by separation of total cell lysates (adjusted to equal amounts of protein) on SDS-PAGE gels. The proteins were then electrotransferred to a PVDF membrane, which was immunoblotted with specific antibodies against phosphor-AKT, acetylate-P53, phosphor-CREB and phosphor-ERK1/2. Figure $4 \mathrm{~A}$ and $4 \mathrm{~B}$ show that both the signaling pathways implicated in NGF-mediated neuritogenesis were activated by Clau $\mathrm{F}$ in a time-dependent manner.

\section{Inhibition of ERK1/2 partially abolishes Clau F-induced neurite outgrowth in PC12 cells}

To investigate whether Clau F promoted neurite outgrowth by activating either the ERK or AKT signaling pathway, PC12 cells were cultured in serum-free medium containing PD98059 (30 $\mathrm{mmol} / \mathrm{L})$ or LY294002 (20 $\mu \mathrm{mol} / \mathrm{L})$, the specific inhibitors of PI3K and ERK, together with Clau F or NGF for $2 \mathrm{~d}$. The 
A
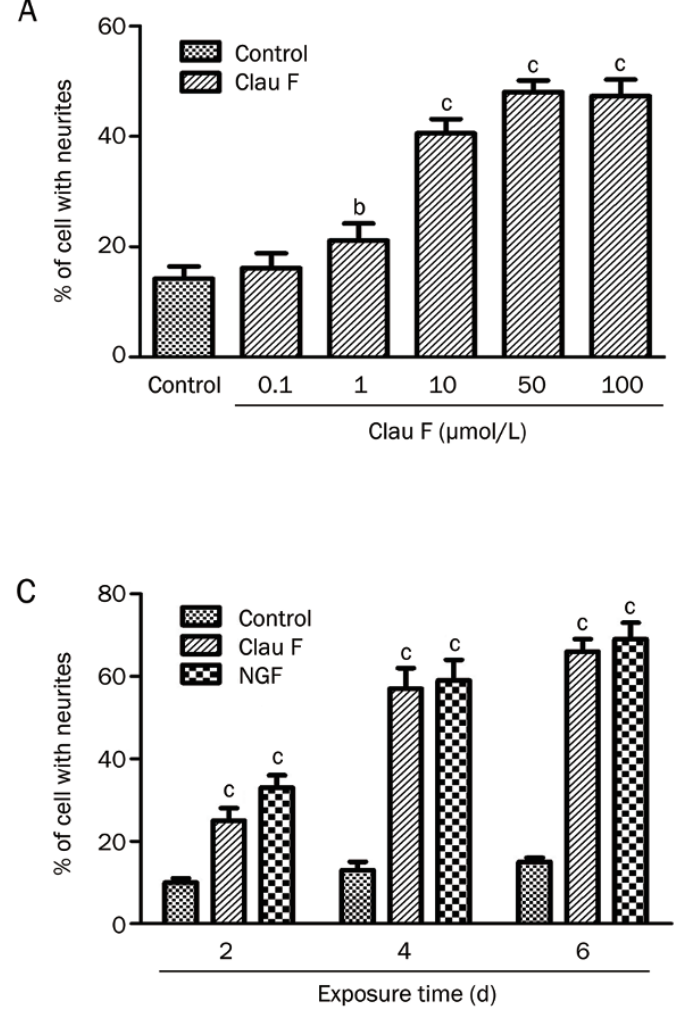

D
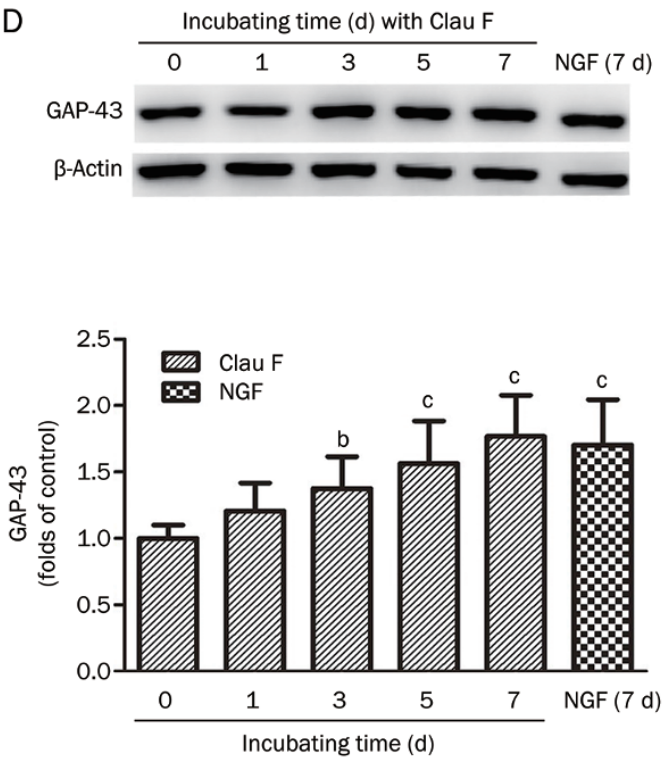

B
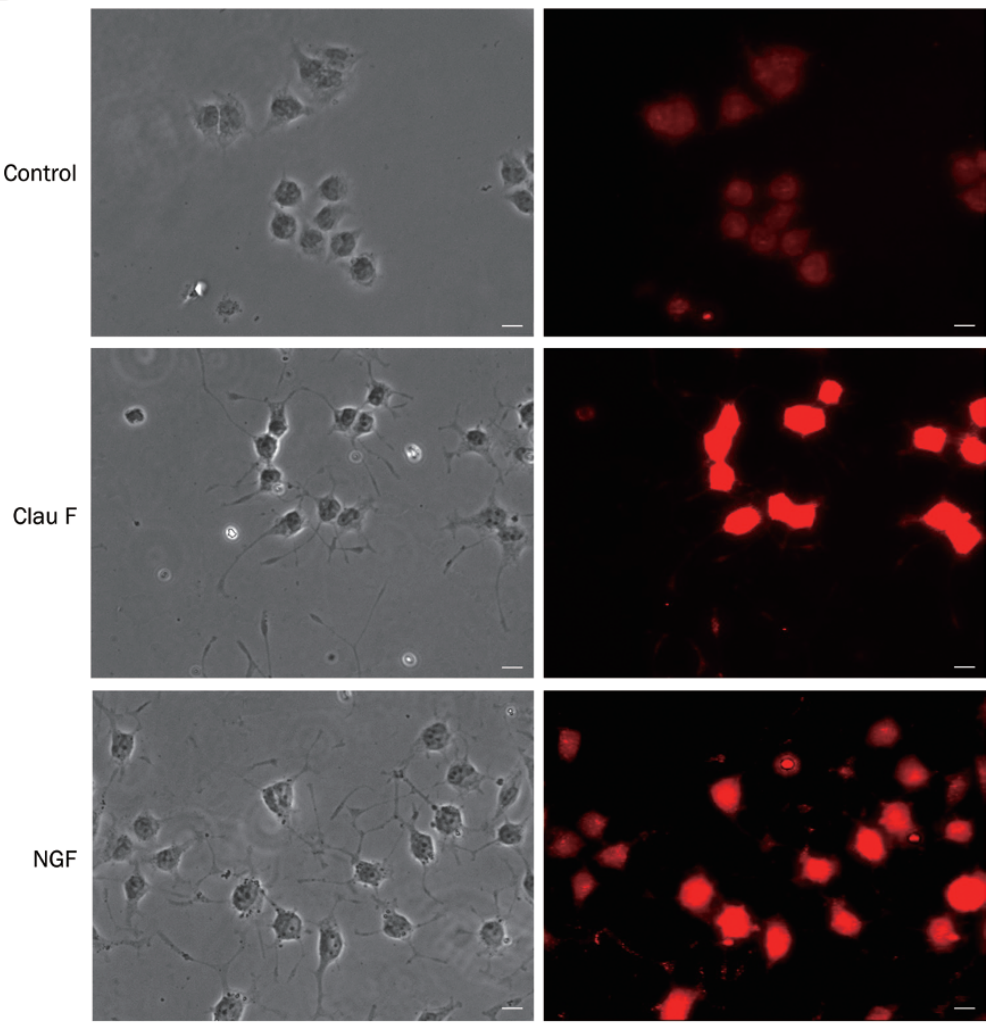

GAP-43
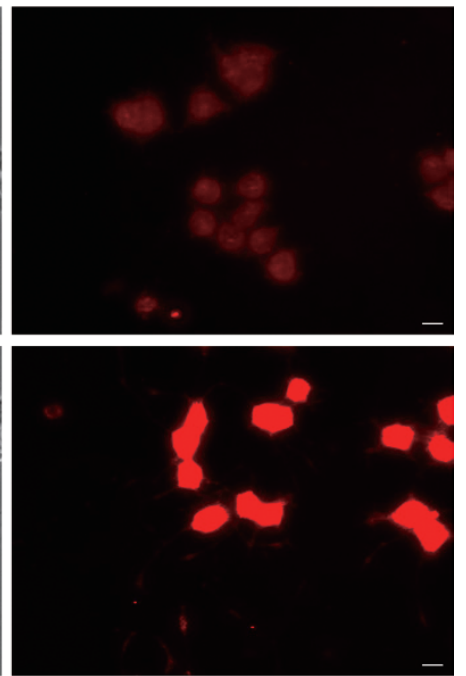
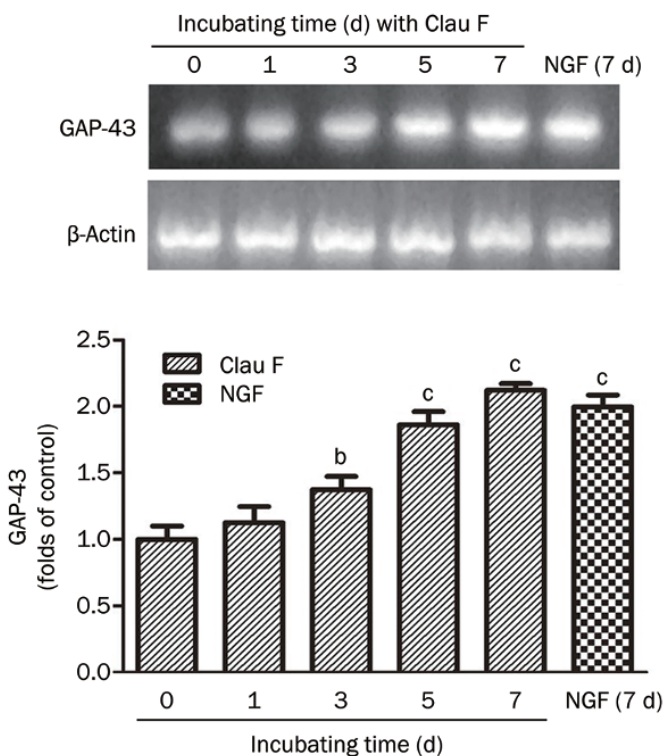

Figure 2. Clau F promoted neurite outgrowth in PC12 cells. Quantitative analysis of band density was performed using three independent Western blotting analyses. (A) Clau F induction of neurite outgrowth was not concentration-dependent, and $10 \mu \mathrm{mol} / \mathrm{L}$ to $100 \mu \mathrm{mol} / \mathrm{L}$ concentrations could initiate neuritogenesis significantly after $2 \mathrm{~d}$ of incubation. (B) The effect of Clau $\mathrm{F}$ on the promotion of neurite outgrowth was examined based on morphology changes and increased expression of GAP-43. (C) The efficiency of neurite outgrowth promoted by Clau F was similar to that of NGF within $6 \mathrm{~d}$ of incubation. (D) The increased expression level of GAP-43 during long-term induction was evidenced both by immunoblotting and RT-PCR. ${ }^{\mathrm{b}} P<0.05,{ }^{\mathrm{c}} P<0.01$ vs control. $\beta$-Actin protein was used as a loading control.

percentage of cells with neurites was determined $2 \mathrm{~d}$ after the treatment. Figure 5A shows that cells incubated with PD98059 and Clau F (c) completely lost their neurite outgrowth ability, whereas a $14.4 \%$ attenuation was observed under Clau F 
A
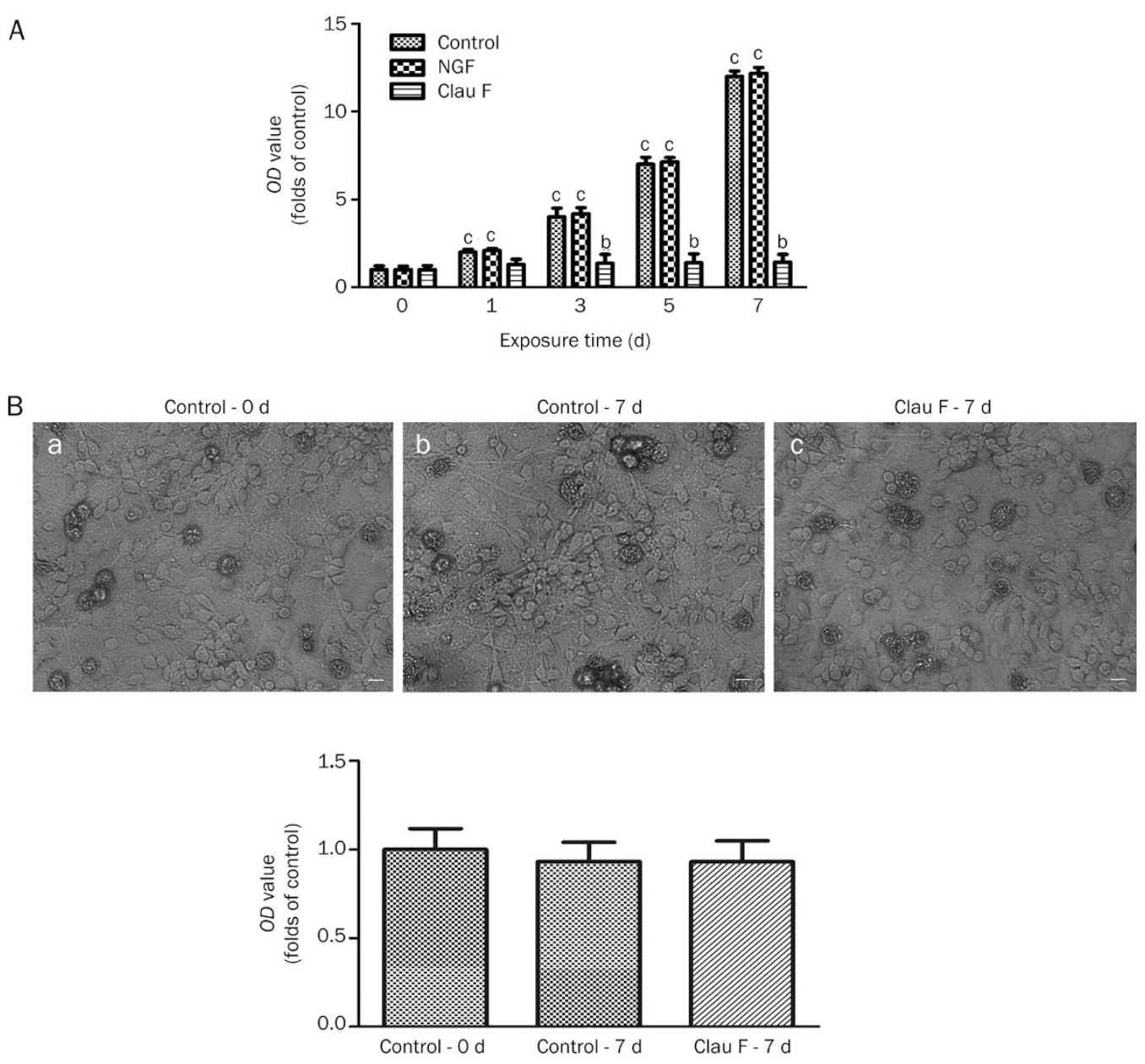

Figure 3. Clau F restrained cell proliferation without causing cell death. After treatment with different agents, the cells were incubated with MTT solution for $4 \mathrm{~h}$ and exposed to an MTT-formazan dissolving solution (a mixture of methanol and DMSO). The optical density (OD) was determined using an absorbance microplate reader (Molecular Devices, Toronto, Canada) set at a wavelength of $570 \mathrm{~nm}$. The cell viability was expressed as a percentage of the $O D$ value of the control cultures. (A) After $7 \mathrm{~d}$ of incubation, the control group showed exponential proliferation, whereas Clau $\mathrm{F}$ showed only moderate multiplication. (B) Cytotoxicity test of Clau F $(10 \mu \mathrm{mol} / \mathrm{L})$ on neural cells from the cerebral cortex of newborn SD rats. No significant difference was observed between the control culture at $0 \mathrm{~d}(\mathrm{a})$, the control culture at $7 \mathrm{~d}(\mathrm{~b})$ or the Clau F-treated culture at $7 \mathrm{~d}(\mathrm{c})$. Morphological changes were observed using an inverted microscope. Mean \pm SEM. ${ }^{\mathrm{b}} P<0.05,{ }^{\mathrm{c}} P<0.01$ vs control.

and LY294002 co-treatment (d). However, a block of either one of the NGF signaling pathways did not hinder the proneuritogenesis effect. Only a block of both pathways caused a relative decrease in the percentage of cells with neurites.

To further examine whether the activation of the ERK pathway is critical for neurite outgrowth, the cells were incubated with inhibitors for $1 \mathrm{~h}$. Subsequently, Clau F or NGF was added for an additional hour of co-incubation prior to harvesting total cellular protein. The levels of GAP-43, phosphorCREB, and acetylated p53 were detected by Western blotting. Figure 5B and 5C show that PD098059 caused a 42.6\% decrease in GAP-43, a 75\% decrease in phosphor-CREB, and a $66.7 \%$ decrease in acetylated p53 compared with the Clau F group. LY294002 caused a 6.7\% decrease in GAP-43, a 22.0\% decrease in phosphor-CREB, and a $19.0 \%$ decrease in acetylated p53 compared with the Clau F group. The suppression of Clau F-mediated neuritogenesis by the ERK inhibitor was corroborated by the involvement of the ERK pathway in the neuritogenic effect of this alkaloid.

\section{Discussion}

It has been shown that (-)clausenamide, a compound extracted from the leaves of Clausena lansium, improves learning and memory in animal models of amnesia ${ }^{[15]}$. Clau F, a carbazole alkaloid that is isolated from the stem of Clansium, shows some distinct bioactivity. Interestingly, Clau $\mathrm{F}$ and the endogenous hormone melatonin have similar structures as well as some common bioactivity, such as free radical clearance and anti-proliferation ${ }^{[16,17]}$.

In this study, we investigated the effect of Clau F on neuritogenesis and finally obtained three novel findings: 1) In addition to anti-proliferation, Clau F, as a carbazole alkaloid, also has a role in neuritogenesis; 2) Clau F promotes neurite outgrowth and expression of GAP-43 with an efficacy similar 
A
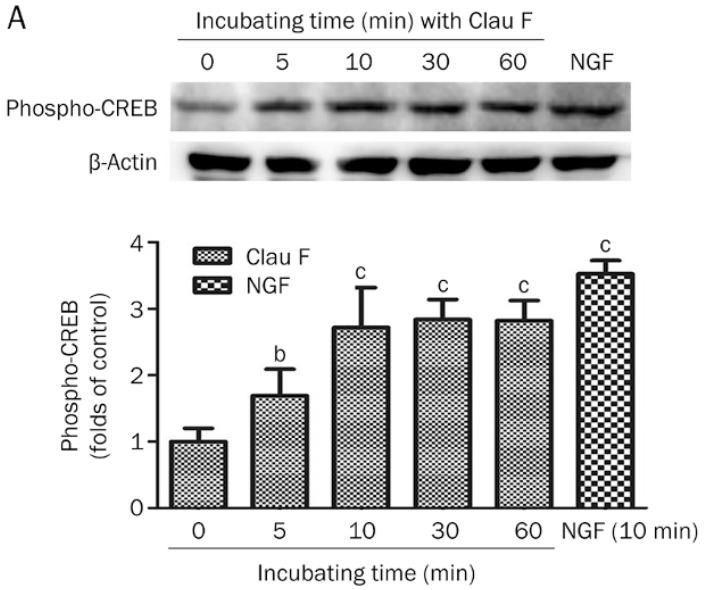

B
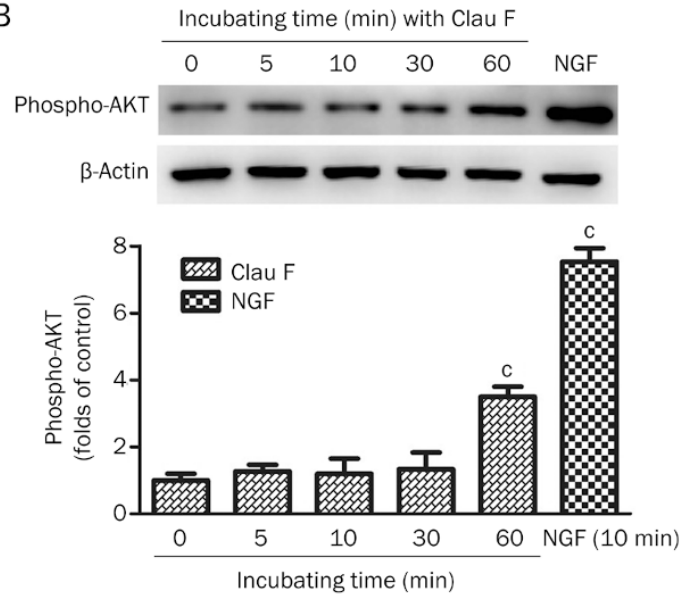
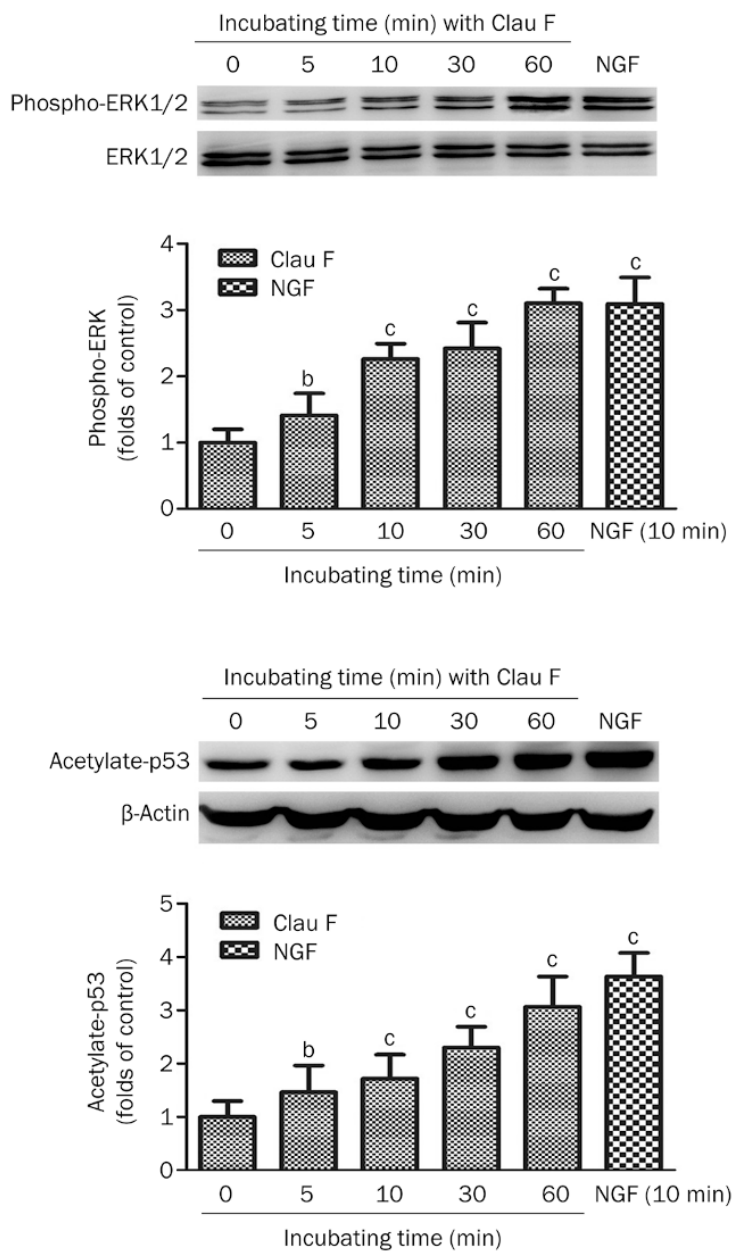

Figure 4. Potentiation of NGF-mediated AKT and ERK signaling was accomplished by Clau F. Quantitative analysis of band density was performed using three independent Western blotting analyses. (A) Clau F stimulated ERK and CREB activation within 60 min of incubation with Clau F. $\beta$-Actin and unphosphorylated ERK1/2 protein were used as loading controls. (B) The phosphorylation level of AKT and the acetylation level of p53 were significantly increased during a 60 -min incubation with Clau F. $\beta$-Actin protein was used as a loading control. Mean \pm SEM. ${ }^{b} P<0.05,{ }^{c} P<0.01$ vs control.

to that of NGF; 3) This effect may be related to the sustained activation of the MAPK-ERK but not the PI3K-AKT signaling pathway.

Undifferentiated PC12 cells did not sprout neurites (less than $1 \%$ ). In our neural model, NGF-differentiated cells produced neurites over a time course, which was in agreement with previous studies ${ }^{[14]}$. Using the percentage of neuritebearing cells as a marker, we found that there was no significant difference between Clau F and NGF with respect to neurite outgrowth promotion.

GAP-43 is a neuron-specific protein, and its synthesis and fast axonal transport are elevated during development and nerve regeneration ${ }^{[18,19]}$. Depletion of GAP-43 markedly alters the morphology of neurites and growth cones ${ }^{[20]}$. Spatial and temporal changes in GAP-43 content and localization in the cell are positively correlated with transmitter release, cytoskeletal regulation and cell growth activated by neurotrophic factors such as $\mathrm{NGF}^{[21-23]}$. Increased expression of GAP-43 was observed in several previous studies when neurite outgrowth was promoted in neural cells under various conditions ${ }^{[24]}$. In contrast, increased expression of GAP-43 has not been reported following central nervous system (CNS) injury in higher vertebrates ${ }^{[2,26]}$.

To further confirm the neurite outgrowth-promoting effect of Clau F, we observed morphological changes in PC12 cells using a fluorescence microscope. As shown in Figure 2B, GAP-43 (red color) was increased substantially in cells incubated with Clau F. We then extended the Clau F incubation time to $7 \mathrm{~d}$ and examined the total expression of GAP-43 by immunoblotting and RT-PCR. As shown in Figure 2D, the levels of GAP-43 protein and mRNA continuously increased for $7 \mathrm{~d}$. There was no significant difference between the Clau F group and the NGF group with respect to the level of GAP-43 expression by $7 \mathrm{~d}$, suggesting that Clau $\mathrm{F}$ has a significant proneuritogenesis effect.

Molecules with similar chemical structures tend to have similar bioactivities. For example, melatonin and carbazole alkaloid both inhibit cell proliferation. However, it was unclear 
A
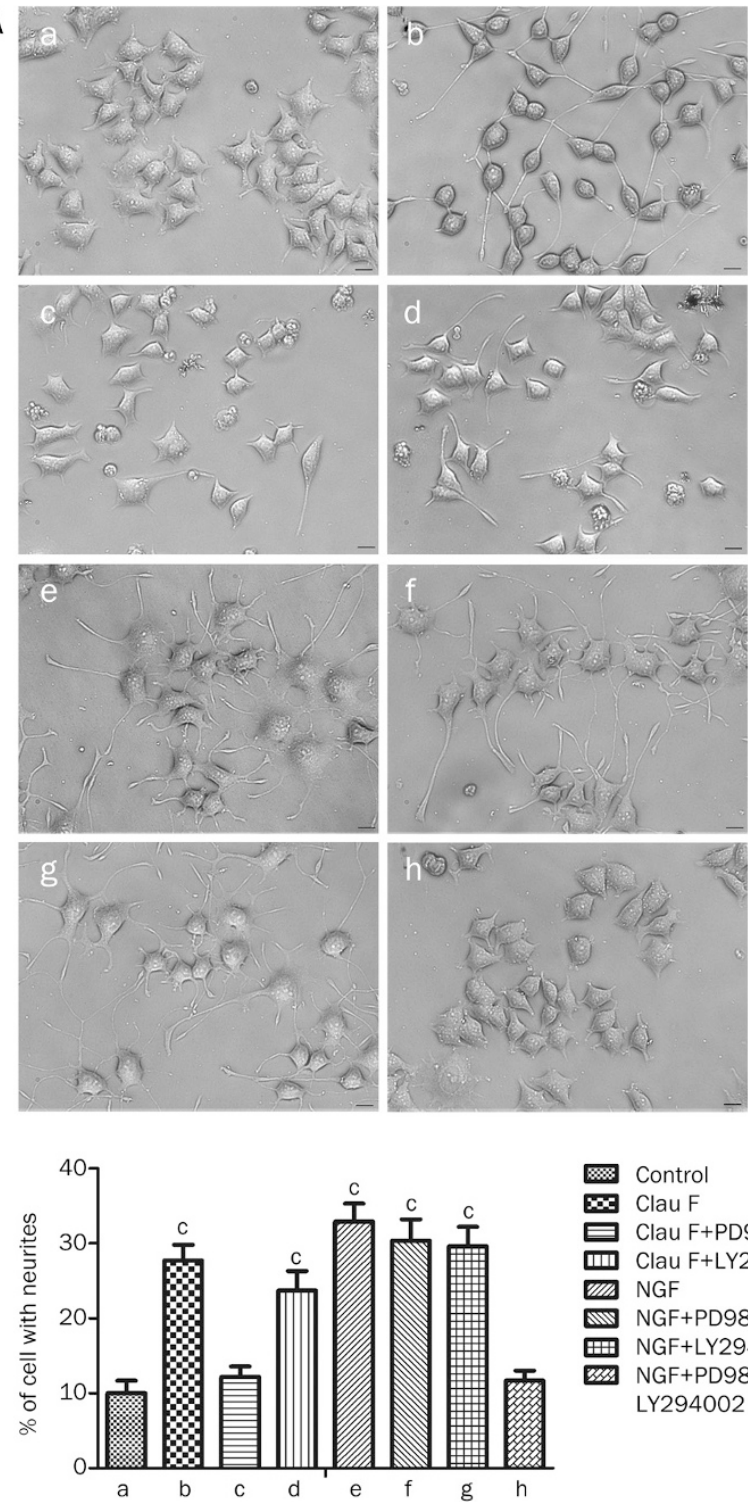

B
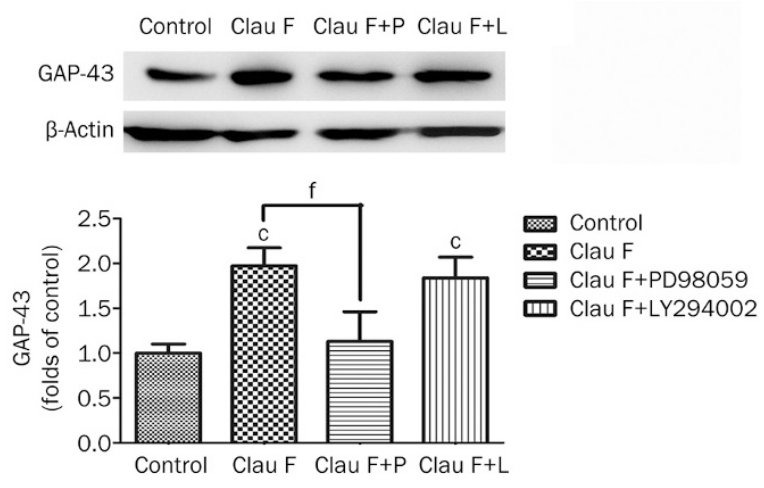

C
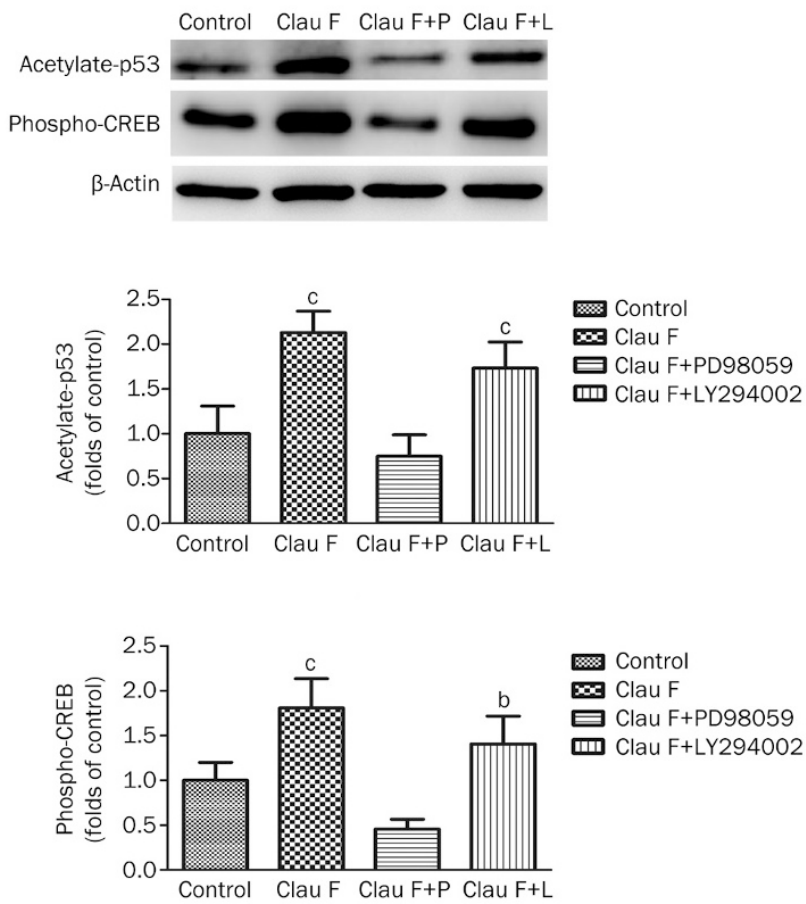

Figure 5. Clau F induced neurite outgrowth by activating the ERK pathway, but not the AKT pathway, in PC12 cells. Quantitative analysis of band density was performed using three independent Western blotting analyses. (A) In the Clau F group (b-d), the ERK-specific inhibitor PD98059 completely abolished neurite outgrowth, whereas the PI3K-specific inhibitor LY294002 only reduced neurite outgrowth by 14.4\%. In the NGF group (e-h), single treatment with PD98059 or LY294002 did not cause significant changes in the ratio of neurite outgrowth; only co-treatment with PD98059 and LY294002 successfully abolished the neurite outgrowth promoted by NGF. Morphological changes were observed with an inverted microscope. (B) The expression of GAP-43 was significantly decreased in the PD98059 group, whereas it was only attenuated by $13 \%$ in the LY294002 group. (C) The levels of phospho-CREB and acetylated p53 were significantly decreased by the ERK-specific inhibitor PD98059. The PI3K-specific inhibitor LY294002 decreased the level of phospho-CREB by $13.3 \%$ and acetylated $p 53$ by $19.0 \%$. Mean \pm SEM. ${ }^{b} P<0.05,{ }^{c} P<0.01$ vs control. $\beta$-Actin protein was used as a loading control.

whether Clau F inhibited cell proliferation through regulation of the cell cycle or through cytotoxicity, ultimately leading to cell death. We explored this further to evaluate the safety of Clau $\mathrm{F}$ and to better understand its exact mechanism of action. We used neural cells from newborn SD rats as the cell model because they exhibit a low level of proliferation and they are sensitive to cytotoxicity. After $7 \mathrm{~d}$ of treatment with Clau F, we subjected the cells to the MTT assay and found that there was no significant difference between Clau F and the control groups (examined at $1 \mathrm{~d}$ and $7 \mathrm{~d}$ of culture). This result suggested that Clau F can control cell proliferation through the regulation of certain cell cycle signaling pathways, eventually leading to cell death, and its effect is not due to non-selective cytotoxicity.

NGF activates different intracellular signaling pathways leading to neurite outgrowth, and it is widely accepted that sustained activation of the ERK signaling pathway is critical in neuronal differentiation. 
To examine whether the Clau F-mediated neurite outgrowth effect is involved in the activation of these kinases, we first determined the phosphorylation state of ERK1/2 in PC12 cells after different durations of treatment. As shown in Figure 3A, a noticeable increase in ERK1/2 was observed 30 min after treatment, and a maximal effect was obtained $1 \mathrm{~h}$ after treatment. The degree of phosphorylation of ERK was comparable to that obtained by treatment with NGF. It has been shown that both the magnitude and longevity of MAPK activation govern the nature of the cellular response ${ }^{[27]}$. In PC12 cells, prolonged activation of ERK caused by NGF induces differentiation, whereas transient activation stimulates proliferation. It therefore seems plausible that the late and prolonged activation of ERK1/2 followed by the activation of p53 and CREB, together with the proliferation inhibition induced by Clau F, could be responsible for the neuritic initiation ${ }^{[28]}$.

It has been reported that the increased acetylation of p53 is responsible for its increased binding to the promoters of prooutgrowth genes including CREB, GAP-43, and NGF ${ }^{[29,30]}$. As shown in Figure 3B, acetylation of p53 induced by Clau F was significantly increased compared with the control group. Activation of the PI3K-AKT or MAPK-ERK pathway subsequently induces p53 acetylation, which would stimulate neural outgrowth and cause cell cycle arrest in PC12 cells ${ }^{[31-33]}$. Thus, we investigated the effect of Clau F on the PI3K-AKT pathway by measuring the phospho-AKT (Ser473) level, which was significantly different between the two groups with respect to both activation time course and intensity after treatment. Blockage of the AKT pathway with an AKT-specific inhibitor, LY294002, inhibited Clau F-induced neuritogenesis by $14.4 \%$. However, although both pathways must be blocked to abolish the pro-neuritogenesis effect of NGF, the ERK-specific inhibitor PD98059 completely blocked the neurite outgrowth effect promoted by Clau F.

To further examine whether activation of the ERK pathway is critical for Clau F-induced neurite outgrowth, GAP43 protein levels were measured by Western blotting analysis. PD098059 treatment decreased the expression of GAP-43 to a level similar to that of the control group, whereas LY294002 only slightly decreased GAP-43 (by 6.7\%). A relatively low level of GAP-43 indicated the loss of neurite outgrowth ability in the group incubated with both PD98059 and Clau F, which further demonstrated the critical role of the ERK signaling pathway in neuritogenesis promoted by Clau F. Moreover, PD098059 significantly inhibited the acetylation of p53 (by $66.7 \%$ ) and the phosphorylation of CREB (by 75\%). LY294002 only decreased CREB phosphorylation by $22.0 \%$ and p53 acetylation by $19.0 \%$. The above results indicate that the ERK pathway is critical for the increase in acetylated p53, which in turn confers the neuritogenesis promoted by Clau F.

In summary, Clau F displays neuritogenic activity with an efficacy similar to that of NGF in PC12 cells. We have also provided evidence to suggest that NGF and Clau F-induced neurogenesis are mediated through distinct mechanisms. Whereas NGF-induced neurogenesis is involved in both MAPK-ERK and PI3K-AKT pathways, Clau F-induced neurite outgrowth selectively depends on the activation of ERK but not AKT.

More recent studies have shown that Clau $\mathrm{F}$ can increase the mRNA level of NGF and the expression level of tyrosine hydroxylase $(\mathrm{TH})$. This suggests that Clau F might play a role in promoting differentiation. Our future studies will focus on the precise mechanisms and potency of the pro-differentiation and proliferation inhibition effects induced by Clau $\mathrm{F}$ in neural stem and progenitor cells.

\section{Acknowledgements}

This work was supported by the National Natural Science Foundation of China (Grant No 81274122, 81273629, and 81001487), Special Purpose for New Drug Development (2012ZX09301002-004), Studies on the Structure and Function of Bioactive Substances from Natural Medicines (IRT1007), the Beijing Natural Science Foundation (7131013), and the Research Fund for the Doctoral Program of Higher Education of China (20121106130001).

\section{Author contribution}

Yin-zhong MA performed most of the experiments; Shi-feng CHU analyzed the data; Jin-feng HU and Yin-zhong MA prepared the manuscript; Jing-wei LI and Wen-bin HE assisted in the experiments; and Nai-hong CHEN and Na NING designed the study.

\section{References}

1 Roberson ED, Mucke L. 100 years and counting: prospects for defeating Alzheimer's disease. Science 2006; 314: 781-4.

2 Egleton RD, Davis TP. Bioavailability and transport of peptides and peptide drugs into the brain. Peptides 1997; 18: 1431-9.

3 Maggini M, Vanacore N, Raschetti R. Cholinesterase inhibitors: drugs looking for a disease? PLoS Med 2006; 3: e140.

4 Pollack SJ, Harper SJ. Small molecule Trk receptor agonists and other neurotrophic factor mimetics. Curr Drug Targets CNS Neurol Disord 2002; 1: 59-80.

5 Aebischer P, Ridet J. Recombinant proteins for neurodegenerative diseases: the delivery issue. Trends Neurosci 2001; 24: 533-40.

6 Liu GT, Li WX, Chen YY, Wei HL. Hepatoprotective action of nine constituents isolated from the leaves of Clausena lansium in mice. Drug Dev Res 1996; 39: 174-8.

7 Zoubaa S, Konrad R, Piontek G, Schlegel J. Inhibition of NGF-induced neurite outgrowth of rat pheochromocytoma cells (PC12) following administration of dioxyamphetamine. Neurosci Lett 2010; 476: 113-8.

8 Friedman WJ, Greene LA. Neurotrophin signaling via Trks and p75. Exp Cell Res 1999; 253: 131-42.

9 Zhao Q, Li C, Yang J, Zhang D. Chemical constituents of Clausena lansium. Zhongguo Zhong Yao Za Zhi 2010; 35: 997-1000.

10 Shi GX, Rehmann H, Andres DA. A novel cyclic AMP-dependent EpacRit signaling pathway contributes to PACAP38-mediated neuronal differentiation. Mol Cell Biol 2006; 26: 9136-47.

11 Greene LA, Kaplan DR. Early events in neurotrophin signalling via Trk and p75 receptors. Curr Opin Neurobiol 1995; 5: 579-87.

12 Das KP, Freudenrich TM, Mundy WR. Assessment of PC12 cell differentiation and neurite growth: A comparison of morphological and neurochemical measures. Neurotoxicol Teratol 2004; 26: 397-406. 
13 Brynczka C, Merrick BA. The p53 transcriptional target gene wnt7b contributes to NGF-inducible neurite outgrowth in neuronal PC12 cells. Differentiation 2008; 76: 795-808.

14 Daniele S, Lecca D, Trincavelli ML, Ciampi O, Abbracchio MP, Martini C. Regulation of PC12 cell survival and differentiation by the new P2Ylike receptor GPR17. Cell Signal 2010; 22: 697-706.

15 Duan W, Zhang J. Effects of $(-),(+)$ clausenamide on anisodineinduced acetylcholine decrease and associated memory deficits in the mouse brain. Yao Xue Xue Bao 1998; 33: 259-63.

16 Li JW, Ning N, Ma YZ, Zhang R, Tan F, Chen NH. Claulansine F suppresses apoptosis induced by sodium nitroprusside in PC12 cells. Free Radic Res 2013; 47: 488-97.

17 Liu L, Xu Y, Reiter RJ. Melatonin inhibits the proliferation of human osteosarcoma cell line MG-63. Bone 2013; 55: 432-8.

18 Benowitz LI, Routtenberg A. A membrane phosphoprotein associated with neural development, axonal regeneration, phospholipid metabolism, and synaptic plasticity. Trends Neurosci 1987; 10: 527-32.

19 Skene JH. Axonal growth-associated proteins. Annu Rev Neurosci 1989; 12: 127-56.

20 Aigner L, Caroni P. Depletion of $43-k D$ growth-associated protein in primary sensory neurons leads to diminished formation and spreading of growth cones. J Cell Biol 1993; 123: 417-29.

21 Lannazzo L. Involvement of B-50 (GAP-43) phosphorylation in the modulation of transmitter release by protein kinase C. Clin Exp Pharmacol Physiol 2001; 28: 901-4.

22 Laux T, Fukami K, Thelen M, Golub T, Frey D, Caroni P. GAP43, MARCKS, and CAP23 modulate $\operatorname{PI}(4,5) \mathrm{P}(2)$ at plasmalemmal rafts, and regulate cell cortex actin dynamics through a common mechanism. J Cell Biol 2000; 149: 1455-72.

23 Takeshita M, Banno Y, Nakamura M, Otsuka M, Teramachi H, Tsuchiya $\mathrm{T}$, et al. The pivotal role of intracellular calcium in oxaliplatin-induced inhibition of neurite outgrowth but not cell death in differentiated PC12 cells. Chem Res Toxicol 2011; 24: 1845-52.
24 Larsson C. Protein kinase $\mathrm{C}$ and the regulation of the actin cytoskeleton. Cell Signal 2006; 18: 276-84.

25 Skene JH, Willard M. Axonally transported proteins associated with axon growth in rabbit central and peripheral nervous systems. J Cell Biol 1981; 89: 96-103.

26 Snipes GJ, Costello B, McGuire CB, Mayes BN, Bock SS, Norden JJ, et al. Regulation of snecific neuronal and non-neuronal proteins during development and following injury in the rat CNS. Prog Brain Res 1987; 71: 155-75.

27 Kao S, Jaiswal RK, Kolch W, Landreth GE. Identification of the mechanisms regulating the differential activation of the MAPK cascade by epidermal growth factor and nerve growth factor in PC12 cells. J Biol Chem 2001; 276: 18169-77.

28 Xing J, Kornhauser JM, Xia Z, Thiele EA, Greenberg ME. Nerve growth factor activates extracellular signal-regulated kinase and p38 mitogen-activated protein kinase pathways to stimulate CREB serine 133 phosphorylation. Mol Cell Biol 1998; 18: 1946-55.

29 Costello B, Meymandi A, Freeman JA. Factors influencing GAP-43 gene expression in PC12 pheochromocytoma cells. J Neurosci 1990; 10: $1398-406$.

30 Tedeschi A, Di Giovanni S. The non-apoptotic role of p53 in neuronal biology: enlightening the dark side of the moon. EMBO Rep 2009; 10: 576-83.

31 Emamian ES, Hall D, Birnbaum MJ, Karayiorgou M, Gogos JA. Convergent evidence for impaired AKT1-GSK3beta signaling in schizophrenia. Nat Genet 2004; 36: 131-7.

32 Kita Y, Kimura KD, Kobayashi M, Inara S, Kaibuchi K, Kuroda S, et al. Microinjection of activated phosphatidylinositol-3 kinase induces process outgrowth in rat PC12 cells through the Rac-JNK signal transduction pathway. J Cell Sci 1998; 111: 907-15.

33 Mark MD, Storm DR. Antiproliferative activity of $\mathrm{KCl}$ contributes to EGF-induced neurite outgrowth in PC12 cells. Neurosci Lett 1997; 230: 73-6. 